\title{
Developmental differences in concept transfer as a function of variability of irrelevant features during acquisition
}

\author{
LORRAINE A. LOW, ELLEN COSTE, and CYNTHIA KIRKUP \\ Framingham State College, Framingham, Massachusetts 01701
}

\begin{abstract}
The effect of variability of irrelevant information on concept transfer as a function of age was investigated. The pictorial stimuli were either designs or toy shelves. The individual features for the designs were geometric figures such as a diamond, trapezoid, and so on, and the toy shelves consisted of simple figures such as a dog or plant. The different shapes for each figure were determined by the ratio of the horizontal to the vertical axis. The number of figures that varied in shape during acquisition constituted the extent of variability during acquisition. Results indicated that fourth-graders had fewer errors in transfer with increase in variability during acquisition. For the second-graders, the trend was in the opposite direction. The results reflected developmental differences in ability to infer categories from the relationship between stimulus variation and response change.
\end{abstract}

In recent years, it has become more and more recognized that in a concept learning task, the accurate placement of the relevant dimensions does not necessarily imply that the irrelevant dimensions are also appropriately identified. It has been shown (Modigliani, 1971; Modigliani \& Rizza, 1971) that when individuals learn to classify stimuli according to some relevant criteria, subsequent change in irrelevant features results in marked deterioration in category recognition. One acquisition variable that has been shown to facilitate transfer to new instances is the increase in number of different instances presented during acquisition (Homa \& Chambliss, 1975; Homa, Cross, Cornell, Goldman, \& Schwartz, 1973; Homa \& Vosburgh, 1976).

Explanations for improved transfer with the increase in the number of different acquisition instances have involved the partitioning of various stimulus components into relevant and irrelevant sources (Homa \& Chambliss, 1975; Homa \& Vosburgh, 1976). The broadening of transfer with the increase in the number of instances is attributed to the increased opportunity to identify these various components. Three components have been suggested. First, idiosyncratic stimuli are those irrelevant stimuli that vary within a category. When a red, a green, and a blue triangle are presented, for example, the dimension of color, because of its variability, is idiosyncratic and irrelevant to the concept of triangle.

The authors thank David Meacham for his assistance in the collection of the data. The authors also wish to thank the administrators, teachers, and students of the following Massachusetts elementary schools for their participation: Miller School, Holliston; Saint Bridget's School, Framingham; Brown School and Schofield School, Wellesley; and Clyde Browne School, Memorial School, and Memorial School Annex, Millis. Requests for reprints should be sent to Lorraine A. Low, Psychology Department, Framingham State College, Framingham, Massachusetts 01701.
Second, common irrelevant stimuli do not vary within or between categories and are detected only when different categories are discriminated. If blue triangles and blue squares are presented, for example, blue is common and irrelevant to the triangle and square categories. Its irrelevancy is only detected when at least two categories are compared. Third, common and distinctive features are those components that are relevant to categorization (triangles and squares in the above example). It has been emphasized (Homa \& Chambliss, 1975) that the idiosyncratic stimuli are easier to identify than common irrelevant stimuli because the former do vary within category. As a consequence, these stimuli are more easily discriminated as irrelevant.

It is implicitly assumed in the above model that the information necessary for category placement is present in the various stimulus components and needs only to be detected. Accordingly, the function of increased variation in acquisition instances is to provide the opportunity for correctly identifying these various components. It is argued here that the irrelevant components of a stimulus complex must not only be detected but also be interpreted as irrelevant so that they are eliminated from further consideration for class inclusion. In order to correctly identify irrelevant stimuli it is necessary to infer irrelevancy from the lack of correspondence between stimulus variation and response classification. For example, the continuous change in idiosyncratic stimuli and the corresponding invariance of the response must somehow be learned to be interpreted as irrelevancy of these stimulus components. Similarly, the constancy of certain stimulus components with variation in category naming must also be interpreted as the irrelevancy of these common stimuli to category inclusion. In the present view, it is not the variation or lack of variation in stimuli that determines 
the irrelevancy, but rather the interpretation of the change relative to the category response.

If the partitioning of the stimuli into relevant and irrelevant components is not merely a matter of detection but rather requires learning and experience, then this ability should be age related. Older children should benefit more from variation of irrelevant stimuli than should younger ones in appropriately partitioning the stimulus complex. Therefore older children should show better category transfer with increase in idiosyncratic stimuli, defined by variation in irrelevant dimensions. It is assumed that younger children have not learned to interpret this lack of correspondence as irrelevancy. Rather, variability has a more salient effect for younger than for older children (Odom \& Guzman, 1970). The probability that these dimensions will be attended to and included as part of the category is therefore increased. These children should show poorer category transfer with increased variation during acquisition.

A number of previous studies (Low, Coste, \& Kirkup, 1978; Low, Woolverton, \& Lusignan, 1976) have demonstrated that variability of stimulus information during acquisition did differentially influence concept transfer. Older children showed positive transfer, and younger children showed negative transfer, with increase in acquisition variability. In the study by Low et al. (1978), pictures of faces and houses were presented to second-, fourth-, and sixth-graders. Shapes of features for each figure varied. Examples of features were eyes and ears for the face, and roofs and doors for the house.

Idiosyncratic features were those irrelevant features during acquisition which varied orthogonally with each other and with the single relevant feature during acquisition. The number of such idiosyncratic features was one variable in that experiment. During transfer, the previously constant irrelevant features (common and irrelevant) were varied. The relevant features during acquisition were maintained during transfer. The information of interest was the influence of number of idiosyncratic features varied during acquisition on number of transfer errors as a function of age. The trend suggested that variability of irrelevant features during acquisition had a positive influence on concept transfer for older children but resulted in increased error for younger children. However, the results depended on the particular figure (faces or houses).

The present experiment was designed to test the major variables in the experiment by Low et al. (1978). An attempt was made, however, in this experiment to reduce the effects of the particular figures used. In the present experiment, therefore, each figure was composed of aggregates of features. Each feature was in itself an individual complete figure. The use of this type of whole figure accomplished the following: (1) Each figure was independently manipulated and placed with respect to the whole; (2) each feature was equally weighted in terms of its contribution to the entire configuration; and (3) most important, preexperimental habits in identifying the whole as a unique configuration were minimized.

\section{METHOD}

\section{Subjects}

Subjects were second-, fourth-, and sixth-graders, 32 from each grade. They were randomly selected from local elementary schools and randomly assigned to one of four conditions, two variability conditions (Var) and two specific-figure conditions (SF).

\section{Stimulus Material and Design}

The design was similar to the one in the previous experiment (Low et al., 1978). The figures used in this experiment, however, were either geometric shapes (designs) or shelves with toys on them (toy shelves). These figures constituted the SF condition. The 13 features for each stimulus were stenciled in black ink on poster board and uniformly distributed on three lines. Some examples of the features for designs were a heart, a diamond, and a trapezoid, and for toy shelves, a dog, a plant, and a cup. The relevant features for category assignment were an inverted$T$ shape for the designs and a cup for toy shelves. The basic contour of the feature did not vary. The different shapes for each feature were defined by the various ratios of the horizontal to the vertical axis, which were as follows: $2: 1,1: 1$, and $1: 2$. Two of the three shapes for each feature were randomly selected if that feature varied, and one was randomly selected if that feature remained constant during either acquisition or transfer. For a particular trial, in acquisition and in transfer, each irrelevant shape was chosen so that it was orthogonal to the other irrelevant shapes and to the one relevant shape.

The two Var conditions during acquisition were defined by the variation of one and four features (Conditions $V_{1}$ and $V_{4}$, respectively). Thus, for the $V_{1}$ condition, there were two different bilevel features (one relevant and one irrelevant), and for the $\mathrm{V}_{4}$ condition there were five different bilevel features (one relevant and four irrelevant). This resulted in 4 and 32 different acquisition stimuli for Conditions $V_{1}$ and $V_{4}$, respectively.

The treatment conditions were the same for all subjects during transfer. The irrelevant features that varied during transfer were chosen from those features that were constant during acquisition. The number of features varied during transfer was termed the transformation variable, $T$. Thus, $T_{1}, T_{4}$, and $T_{7}$ represented the trials in which one, four, or seven of the previously constant features, respectively, were now varied in transfer. Half of the transfer stimuli contained a new relevant shape; half contained one of the two old relevant shapes. The old and new relevant shapes constituted another transfer variable $(\mathrm{O}, \mathrm{N})$. The new relevant shape was introduced so that the child could respond correctly with the term "neither" if the stimulus did not include the acquisition relevant shapes. The design was a 3 (age) by 2 (Var) by 2 (SF) by $2(O, N)$ mixed factorial with the last two factors within-subjects variables.

\section{Procedure}

The child was informed that the procedure was a game in which each picture was to be placed in one of two categories. For designs, it was "Joe's Design" or "Eddie's Design," and for toy shelves, it was "Joe's Shelf" or "Eddie's Shelf." All possible features were indicated to the child, and he was then informed that only one feature would be relevant to the label. The task was to label the stimulus correctly. The child was informed after each response whether he was correct. All children were run to a criterion of 12 correct out of 15 consecutive acquisition trials. A minimum of 20 trials and a maximum of 40 were given, and those children who did not reach a criterion were eliminated.

During transfer, the three levels of $\mathrm{T}$ and two levels of $\mathrm{O}, \mathrm{N}$ 
were orthogonally combined and randomly assigned to each block of six trials. The child was told that some of the pictures to be presented belonged to Joe or Eddie and that he was to respond as he did in acquisition. However, there were pictures that belonged to neither Joe nor Eddie, and for those pictures, he was to say "neither." A total of 36 trials was presented in transfer.

\section{RESULTS}

\section{Acquisition}

The score was the total number of errors in identifying the relevant categories. An analysis of variance was performed on the 3 (age) by 2 (SF) by 2 (Var) betweensubjects factorial design. None of the main effects interactions was statistically significant.

\section{Transfer}

The response measure was the total number of errors in which the old stimulus (Joe or Eddie) was identified as new. The results of Experiment 2 are given in Figure 1. Age was statistically significant $[F(4,84)=3.87, p<.05]$. The means of $3.15,3.78$, and 1.31 for Grades 2,4 , and 6 , respectively, indicate decreasing error with age. A Tukey test for specific comparisons revealed no significant difference between the second- and fourth-graders but a significant difference between the second- and sixth-graders and between the fourth- and sixth-graders ( $\mathrm{p}<.01$ for both). The effects of $\mathrm{T}$ were statistically significant $[F(2,168)=13.50, p<.01]$. It is evident from inspection of Figure 1 that errors increased linearly with $\mathrm{T}$. The Age by Var interaction was statistically significant $[F(2,84)=3.19, p<.05]$. A Tukey test for specific comparisons yielded a difference for the secondgraders that fell just short of significance. For the fourth-graders, the difference was statistically significant $(p<.05)$. The differences for the sixth-graders were small and nonsignificant. In addition, in the $\mathrm{V}_{1}$ condition, the second-graders produced significantly fewer errors than the fourth-graders $(\mathrm{p}<.01)$.

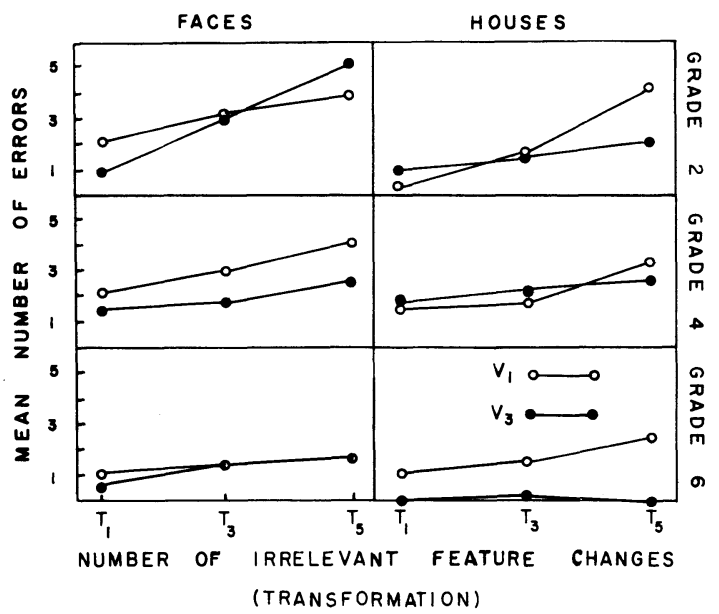

Figure 1. Mean number of errors in calling the "old" categories "neither" as a function of grade level (age), acquisition variability (Var), and degree of transformation during transfer (T) for the combined specific figures (SF).

\section{DISCUSSION}

The findings for the fourth-graders have strong implications for concept acquisition. It has previously been proposed that age differences in concept learning are associated with differences in the ability to define the correct category either by means of verbal labeling (Kendler \& Kendler, 1962) or through attention to correct dimensions (Zeaman \& House, 1963). The present findings do indicate that an important aspect of learning the correct category is the elimination from consideration of the irrelevant dimensions. The variables that enhance this discrimination are not necessarily the ones involved in the correct placement of relevant stimuli. Although the second-graders were able to correctly identify the relevant dimension during acquisition, increased variability resulted in an increase in transfer errors. The fourth-graders, on the other hand, were able to utilize information from the variability of the irrelevant dimensions in reducing the tendency to call the old categories "neither" during transfer.

The lack of differences for the sixth-graders in the $V_{1}$ and $\mathrm{V}_{4}$ conditions might have reflected the ease with which this simple affirmative concept was learned. It is possible that when the task is relatively easy, variables that differentially determine the elimination of the irrelevant information have no effect. With a more difficult task, perhaps, such as the acquisition of a disjunctive or conditional concept, the effects of variability would operate for the oldest children as well.

The unexpected findings that the second-graders were superior to the fourth-graders in the $V_{1}$ condition remains to be explained. The fourth-graders were observed to scan the entire set of stimuli more than the second-graders in the $V_{1}$ condition. Since the figures were composed of separate whole configurations that remained in a constant position throughout the experiment, identification of the correct feature was possible with a minimum of scanning. If there is less scanning by the second-graders, then change from acquisition to transfer in the common features could go unnoticed by them. On the other hand, if the fourthgraders scan more in the $V_{1}$ condition, then the change from acquisition to transfer would be more likely to be noticed by these children, resulting in a greater number of errors.

The present proposal of age-related differences in interpretation of variability of information need not be limited to the processing of visual information. Clark (1973) has suggested that older children acquire more precise word meanings because they are better able to extract just those criterial semantic features that constitute word definition. The present proposal would stress the role of variability of semantic usage in differentially aiding older children in extracting those common and distinctive semantic features that give a word its meaning. It would be expected, for example, that in learning the definition of a new word, older children would benefit more than younger ones from an increase in the variety of sentences that contain the word.

\section{REFERENCES}

Clark, E. What's in a word? On the child's acquisition of semantics in his first language. In T. E. Moore (Ed.), Cognitive development and the acquisition of language. New York: Academic Press, 1973.

Нома, D., \& Chамвliss, D. The relative contributions of common and distinctive information on the abstraction from illdefined categories. Journal of Experimental Psychology: Human Learning and Memory, 1975, 1, 351-359.

Homa, D., Cross, J., Cornell, D., Goldman, D., \& Schwartz, S. Prototype abstraction and classification of new instances as a function of number of instances defining the prototype. Journal of Experimental Psychology, 1973, 101, 116-122.

Нома, D., \& Vosburgh, R. Category breadth and the abstraction of prototypical information. Journal of Experimental Psychology: Human Learning and Memory, 1976, 2, 322-330. Kendeer, H. H., \& Kendier, T. S. Vertical and horizontal 
processes in problem solving. Psychological Review, 1962, 69, $1-16$.

Low, L. A., Coste, E., \& Kirkup, C. Concept transfer as a function of age and variability of irrelevant features during acquisition. Bulletin of the Psychonomic Society, 1978, 12, 393-395.

Low, L. A., Woolverton, M., \& Lusignan, R. Influence of variability of irrelevant features on concept transfer in children. Bulletin of the Psychonomic Society, 1976, 8, 422-424.

Modigliani, V. On the conservation of simple concepts: Generality of the affirmation rule. Journal of Experimental Psychology, 1971, 87, 234-240.

Modigliani, V., \& Rizza, J. P. Conservation of simple concepts as a function of deletion of irrelevant attributes. Journal of Experimental Psychology, 1971, 90, 280-286.

Odom, R. D., \& Guzman, R. D. Problem solving and the perceptual salience of variability and constancy: A developmental study. Journal of Experimental Child Psychology, 1970, 9, 156-165.

Zeaman, D., \& House, B. J. The role of attention in retardate discrimination learning. In N. R. Ellis (Ed.), Handbook of mental deficiency. New York: McGraw-Hill, 1963.

(Received for publication April 1, 1980.) 